\title{
Visual texture perception and Fourier analysis
}

\author{
LEWIS O. HARVEY, Jr., and MARTIN J. GERVAIS \\ University of Colorado, Boulder, Colorado 80309
}

\begin{abstract}
The relationship between the Fourier spectra of visual textures (represented by four hypothetical visual channels sensitive to spatial frequencies) and the perceptual appearance of the textures was investigated. Thirty textures were synthesized by combining various spatial frequencies of different amplitudes. Twenty subjects grouped the textures into $2,3,4$, and 5 groups based on the similarity of their appearance. The groupings were analyzed by means of linear discriminant analysis using the activity of the four channels as predictor variables. The groupings were also examined by. multidimensional scaling, and the resulting stimulus configuration was canonically correlated with the channel activity. The results of both analyses indicate a strong relationship between the perceptual appearance of the textures and their Fourier spectra. These findings suport a multiple-channel spatial-frequency model of perception.
\end{abstract}

Beginning with the landmark paper of Campbell and Robson (1968), a large body of experimental evidence has been published supporting the conclusion that for threshold detection of visual stimuli, the visual system behaves as if it were composed of a number of independent channels, each sensitive to a limited range of spatial frequencies and orientations (Blakemore \& Campbell, 1969; Graham, 1972; Graham \& Nachmias, 1971; Graham \& Rogowitz, 1976; Kelly, 1975, 1976; Kelly \& Magnuski, 1975; Mostafavi \& Sakrison, 1976; Sachs, Nachmias, \& Robson, 1971; Schnitzler, 1976a, 1976b; Spitzberg \& Richards, 1975; Stromeyer \& Julesz, 1972; Stromeyer \& Klein, 1975; Sullivan, Georgeson, \& Oatley, 1972), although there seems to be some contradictory evidence as well (Henning, Hertz, \& Broadbent, 1975; Legge, 1976; Nachmias, Sansbury, Vassilev, \& Weber, 1973). Sekuler (1974) provides a nice review of the psychological and physiological literature. It is fair to say, at the least, that the spatial-frequency content of a visual stimulus, as represented by a twodimensional Fourier transform of the spatial distribution of luminance on the retina, is an important determinant of the detection of the stimulus by the human visual system.

Figure 1 illustrates a generalized independentchannel model of visual detection, adapted from Schnitzler (1976a, 1976b). The major components of the model are the stimulus, the ocular optics, the retina, and several independent channels; each

Reprints may be obtained by writing to Lewis O. Harvey, Jr., Department of Psychology, University of Colorado, Boulder, Colorado 80309 . The data analysis and preparation of this manuscript were partially supported by a grant from the National Science Foundation (BNS76-04652) to the first author. We thank Jonathan $O$. Roberts for several suggestions concerning the analysis of the data. We also thank Professor Whitman Richards, Department of Psychology, Massachusetts Institute of Technology, for the preparation and loan of the stimulus textures. followed by a separate detector mechanism. Phase is ignored in this model since it plays little role in detection. Although we really must use the twodimensional Fourier transform of stimuli, for convenience of graphic representation, only onedimensional transforms are shown in Figure 1. On the left of the diagram, the stimulus is represented by its spatial-frequency amplitude spectrum. An image of the stimulus is formed on the retina and, as a consequence, its spatial-frequency amplitude spectrum is modified by the modulation transfer function (MTF) of the eye's optical system. The light energy falling on the retina is converted into neural activity (labeled "retinal response"), and this activity forms the input to a number of separate channels. Each channel has its own spatial-frequency response (SFR) function, with peak sensitivity that varies in frequency from channel to channel. The output of these separate channels feed to separate and independent detector mechanisms, which make decisions about whether or not the activity in their own channel represents the presence or absence of a visual stimulus. The elements of the model to the right of the detector mechanisms represents the suprathreshold consequences of this model that are examined in this paper.

Much contemporary research has been devoted to psychophysical measures that are used to infer the characteristics of the various elements of the model, especially the characteristic shape of the spatialfrequency-sensitive channels. The bandwidth of the individual channels has been estimated by a variety of psychophysical techniques to be very narrow (Quick \& Reichert, 1975), medium (Blakemore \& Campbell, 1969; Mostafavi \& Sakrison, 1976; Stromeyer \& Julesz, 1972; Stromeyer \& Klein, 1975), or broad (Richards \& Polit, 1974; Spitzberg \& Richards, 1975). If a multiple-channel model such as that shown in Figure 1 is to be taken seriously, it 


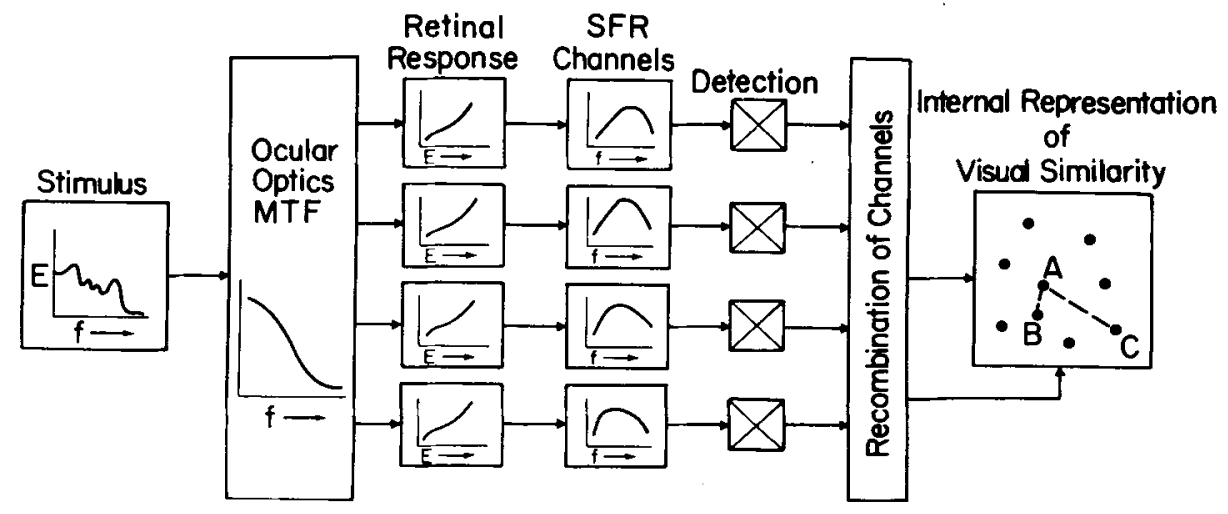

Figure 1. Generalized multiple-channel model of visual detection and perception. (Based on Schnitzler, 1976a, 1976b. See text for details.)

should be predictive of more than just threshold detection; it should be relevant to suprathreshold perception. The current experiment explicitly examines the relationship between the spectrum (as represented by activity in hypothetical neural channels) and the perceptual appearance of the stimuli.

Richards and Polit (1974) generated one-dimensional visual textures by combining vertical sine-wave gratings of various spatial frequencies and modulation amplitudes. The resulting textures were similar to those shown in Figure 2. In a manner analogous to color matching, Richards and Polit had observers match the visual appearance of each texture by manipulating the amplitude of four "primary" spatial frequencies mixed together to form a matching texture $(0.75,2.0,5.0$, and $9.5 \mathrm{cpd}$ were the "primaries" used). The coefficients found experimentally were then subjected to a linear transformation to form four "physiological primaries" or channels. According to these results, if two such textures, regardless of their actual spatial-frequency content, evoke the same amount of activity in the four channels, they should appear identical. Such an appearance match is like a metameric match in color: Two fields have the same color appearance if, regardless of their actual wavelength composition, they cause the same quantal absorption in each of the three cone receptors.

An extension of Richards and Polit's findings is that visual textures which cause similar, but not identical, activity in the four channels will appear more similar than visual textures that evoke widely different patterns of activity in the four channels. The strongest assertion possible is that the perceptual appearance of the visual textures is created by the output of the four channels.

We used two techniques for testing the predicted relationship between visual similarity, on the one hand, and channel activity, on the other. One is based on a linear discriminant analysis (Cooley \& Lohnes, 1971; van de Geer, 1971) of groups of visual textures judged by subjects to appear similar to each other. This type of analysis has been proposed as a model of perceptual processing (Hake \& Rodwan, 1966; Rodwan \& Hake, 1964) and has been successfully used by a number of investigators to infer the kind of stimulus information used by subjects in performing a variety of perceptual tasks (see, for example, Aiken \& Brown, 1971a, 1971b).

The second technique is multidimensional scaling (MDS). [See Shepard (1974) for critical overview of these scaling methods.] The purpose of MDS is to represent a set of stimulus objects as points in an n-dimensional space, such that the distance between points corresponds as closely as possible to subjects' judgments about the similarity of the stimuli (the more similar the stimuli, the closer will be their points in the space). We will refer to this space as a "perceptual" space. If similarity judgments are based on the activity in neural channels, then there should be a high degree of congruence between the perceptual space and the activity in the channels.

We have performed such analyses. We asked subjects to examine $\mathbf{3 0}$ visual textures and to form groups of textures based on the similarity of their appearance. We then analyzed the groupings to relate them to the calculated outputs of three types of channels: Richards and Polit's (1974) four channels, four exponential filter channels (Mostafavi \& Sakrison, 1976), and four Butterworth filter channels (Mostafavi \& Sakrison, 1976). The results of both analyses show that visual similarity of the visual textures is highly dependent on the spatial-frequency content of the textures represented by the activity generated in the four channels and that the Richards and Polit channels are consistently better than the other two types.

\section{METHOD}

\section{Subjects}

Twenty subjects, drawn from the Department of Psychology subject pool, participated in the study in order to partially fulfill the requirements for a general psychology course. They were 
chosen without regard to sex, and all had normal or correctedto-normal vision. The subjects gave their informed consent in accordance with the ethical standards of the University of Colorado and the American Psychological Association.

\section{Stimuli}

Thirty visual textures, similar to those used by Richards and Polit (1974) and generated using the point-probability density method described in that paper and reproduced on Polaroid film, were lent to us by Professor Richards. An advantage of this method of stimulus generation is that the nonlinearity of the photographic process does not affect the linearity of the reproduced waveform. At our viewing distance of $67 \mathrm{~cm}$, these textures subtended $5.0^{\circ} \times 2.25^{\circ}$ of visual angle. The mean luminance of each texture was $25.0 \mathrm{~cd} / \mathrm{m}^{2}$, and the maximum contrast was constant for all textures at .8 . The textures were mounted separately on $14-\times 18-\mathrm{cm}$ pieces of gray (reflectance $=.5$ ) stiff mat board for ease of handling. In order to minimize the effects of the phase of the component frequencies, each texture contained only components whose spatial frequencies were proportional to prime numbers and were not harmonically related to each other. The textures were generated by combining various spatial frequencies, ranging from .3 to $25.6 \mathrm{cpd}$. The spatial frequencies and their amplitudes are given for each texture in Table 1. The spatial frequency of the textures due to the grain created by the pointprobability density method of producing the texture is centered on $38 \mathrm{cpd}$, with a half bandwidth of less than .2 octaves, well outside the range of our highest texture frequency of $25.6 \mathrm{cpd}$. Four of the 30 textures are illustrated in Figure 2.

\section{Channel Models}

Richards and Polit. The weighting coefficients of Richards and Polit's four channels (visibility, low, medium, and high) were

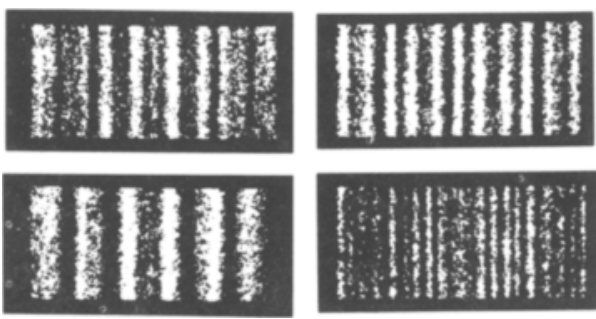

Figure 2. Four examples of visual texture used in the experiment. The horizontal luminance waveforms are created by Fourier synthesis. The probability that a white dot is plotted at any horizontal position is proportional to the waveform amplitude.

taken directly and by interpolation for the specific spatial frequencies used in the current study from Table 2 of their 1974 paper. These channels are not expressed analytically and are shown graphically in Figure 3a.

Exponential channels. The shape of the exponential channels used in the current paper is represented by the function:

$$
\mathbf{H}\left(\mathrm{f}_{\mathrm{r}}\right)=\operatorname{Exp}\left[-1 / 2\left(\frac{\mathrm{f}_{\mathrm{r}}-\mathrm{f}_{\mathrm{g}}}{\mathbf{w}}\right)^{2}\right]
$$

where $H\left(f_{r}\right)$ is the response of the channel to spatial frequency $f_{r}$, $f_{o}$ is the peak frequency of the channel, and $w$ is the bandwidth of the channel. We used a value of $w=f_{0} / 1.8$, the bandwidth found by Mostafavi and Sakrison (1976) for a channel with a peak frequency of $4.5 \mathrm{cpd}$. The center frequencies used in our

Table 1

Spatial Frequencies (SF) in cpd and Amplitudes (A) of Stimulus Textures

\begin{tabular}{|c|c|c|c|c|c|c|c|c|c|c|c|c|c|c|}
\hline \multirow{2}{*}{$\begin{array}{l}\text { Stim- } \\
\text { ulus }\end{array}$} & \multicolumn{2}{|c|}{1} & \multicolumn{2}{|c|}{2} & \multicolumn{2}{|c|}{3} & \multicolumn{2}{|c|}{4} & \multicolumn{2}{|c|}{5} & \multicolumn{2}{|c|}{6} & \multicolumn{2}{|c|}{7} \\
\hline & $\overline{\mathrm{SF}}$ & $\overline{\mathbf{A}}$ & $\overline{\mathrm{SF}}$ & $\overline{\mathbf{A}}$ & SF & $\mathbf{A}$ & $\overline{\mathbf{S F}}$ & $\overline{\mathbf{A}}$ & SF & $\mathbf{A}$ & $\overline{S F}$ & A & SF & A \\
\hline $\begin{array}{l}1 \\
2\end{array}$ & $\begin{array}{l}1.1 \\
4.1\end{array}$ & $\begin{array}{l}.04 \\
4.0\end{array}$ & 1.7 & .13 & 1.9 & .09 & 3.1 & .09 & 4.7 & .04 & & & & \\
\hline 3 & 1.9 & .04 & 3.1 & .09 & 4.1 & .13 & 4.7 & .09 & 6.1 & .04 & & & & \\
\hline 4 & .7 & .14 & 1.9 & .14 & 4.7 & .14 & & & & & & & & \\
\hline 5 & 10.7 & .40 & & & & & & & & & & & & \\
\hline 6 & 3.1 & .10 & 4.1 & .20 & 4.7 & .10 & & & & & & & & \\
\hline 7 & 1.9 & .10 & 3.1 & .20 & 4.7 & .10 & & & & & & & & \\
\hline 8 & 1.1 & .10 & 1.7 & .20 & 1.9 & .10 & & & & & & & & \\
\hline 9 & 1.7 & .40 & & & & & & & & & & & & \\
\hline 10 & 3.1 & .40 & & & & & & & & & & & & \\
\hline 11 & 21.1 & .40 & & & & & & & & & & & & \\
\hline 12 & 1.9 & .04 & 3.1 & .04 & 4.7 & .09 & 6.1 & .09 & 8.3 & .10 & & & & \\
\hline 13 & 25.6 & .40 & & & & & & & & & & & & \\
\hline 14 & 8.3 & .40 & & & & & & & & & & & & \\
\hline 15 & 5.3 & .40 & & & & & & & & & & & & \\
\hline 16 & 1.1 & .04 & 1.9 & .09 & 2.3 & .13 & 3.1 & .09 & 4.7 & .04 & & & & \\
\hline 17 & 1.1 & .10 & 1.3 & .20 & 1.9 & .10 & & & & & & & & \\
\hline 18 & .5 & .06 & .7 & .06 & 1.1 & .06 & 1.9 & .06 & 3.1 & .06 & 4.7 & .06 & 6.1 & .06 \\
\hline 19 & 1.1 & .10 & 1.9 & .10 & 2.3 & .20 & & & & & & & & \\
\hline 20 & .7 & .04 & 1.1 & .09 & 1.3 & .13 & 1.9 & .09 & 3.1 & .04 & & & & \\
\hline 21 & 1.3 & .40 & & & & & & & & & & & & \\
\hline 22 & .5 & .12 & .7 & .04 & 1.1 & .09 & 1.9 & .09 & 3.1 & .04 & & & & \\
\hline 23 & .7 & .10 & .8 & .20 & 1.1 & .10 & & & & & & & & \\
\hline 24 & .9 & .40 & & & & & & & & & & & & \\
\hline 25 & .5 & .40 & & & & & & & & & & & & \\
\hline 26 & .3 & .13 & 1.9 & .13 & 9.1 & .13 & & & & & & & & \\
\hline 27 & .5 & .13 & 3.8 & .13 & 18.2 & .13 & & & & & & & & \\
\hline 28 & .3 & .13 & 1.9 & .13 & 9.1 & .13 & & & & & & & & \\
\hline 29 & .3 & .40 & & & & & & & & & & & & \\
\hline 30 & .5 & .20 & 1.1 & .10 & 1.9 & .10 & & & & & & & & \\
\hline
\end{tabular}


Table 2

Performance of the Discriminant Analyses for the Three Types of Channels

\begin{tabular}{|c|c|c|c|c|}
\hline & \multirow[b]{2}{*}{$\begin{array}{l}\text { Canonical } \\
\text { Correlation }\end{array}$} & \multicolumn{3}{|c|}{ Classification } \\
\hline & & $\begin{array}{c}\text { Percent } \\
\text { CC }^{*}\end{array}$ & $\begin{array}{l}\text { Percent } \\
\text { Correct }\end{array}$ & $\begin{array}{c}\text { Distance } \\
\text { D }\end{array}$ \\
\hline Richards \&.Polit & .629 & 72.5 & 79.1 & 1.707 \\
\hline Random & .348 & 2.5 & 65.8 & .745 \\
\hline$t(78)$ & 10.3 & & 8.14 & 8.87 \\
\hline Butterworth & .576 & 60.0 & 73.3 & 1.509 \\
\hline Random & .367 & 2.5 & 64.5 & .790 \\
\hline$t(78)$ & 7.3 & & 4.62 & 6.37 \\
\hline Exponential & .575 & 57.5 & 75.3 & 1.514 \\
\hline Random & .365 & 5.0 & 63.5 & .785 \\
\hline $\mathrm{t}(78)$ & 6.9 & & 6.83 & 6.35 \\
\hline
\end{tabular}

*Percent CC significant at .05.

analysis were arbitrarily chosen to be $1.0,3.0,5.0$, and $10.0 \mathrm{cpd}$. The resulting four channels are shown in Figure $3 \mathrm{~b}$.

Butterworth channels. The results of Mostafavi and Sakrison (1976) indicate that the shape of the channel centered on $4.5 \mathrm{cpd}$ is better approximated by a Butterworth-type filter represented by the following function:

$$
H\left(f_{r}\right)=\left[1+\left(\frac{f_{r}-f_{o}}{w}\right)^{2}\right]^{-1 / 2}
$$

where $f_{r}, f_{o}$, and $w$ have the same meanings as in Equation 1. The same arbitrary center frequencies of 1.0, 3.0, 5.0, and 10.0 were used with the Butterworth-type channels. The resulting four channels are shown in Figure 3c.

Channel outputs. We assumed that each channel linearly summates the effect of each spatial frequency presented to it. The output of each channel was calculated for each texture by multiplying the amplitude of each spatial-frequency component by the corresponding channel-filter factor and summing the individual responses to achieve a single response for each channel for each texture. As a result, each texture can be represented by three sets of four numbers: The responses of each of the four channels of the Richards and Polit type, the exponential type, and the Butterworth-filter type.

\section{Procedure}

The subjects were tested individually. The 30 stimulus textures were randomly arranged in a stack in front of the subject, who was then asked by the experimenter to arrange the textures into two groups such that the textures within each group "appear as similar as possible" and that the two groups "appear as different as possible." Other than the constraint that the groups should contain roughly equal numbers, no other requirements were made and the subject was free to perform the task in any appropriate manner. Which textures were in which group was recorded by the experimenter. The subject was then asked to form 3, then 4, and then 5 groups of similar appearing textures. The whole procedure was then repeated, with the subjects again forming 2, 3,4 , and 5 groups of textures. The testing time for each subject was approximately $1 \mathrm{~h}$.

\section{RESULTS}

\section{Discriminant Analysis}

Is there a significant relationship between the groupings of textures formed by the subjects and the activity in the four channels of the various models? In order to answer this question, separate discriminant analyses were calculated for the $2,3,4$, and 5 groupings made twice by each subject, using the three different types of channels as predictor variables. The resulting 480 analyses were made using the Statistical Package for the Social Sciences (SPSS) (Nie, Hull, Jenkins, Steinbrenner, \& Bent, 1975) on the University of Colorado Computing Center's dual CDC-6400 computer system. The results indicate a relationship between the channel activity and the perceptual groupings and that the Richards and Polit (1974) channels consistently bore a stronger relationship than did the other two types of channels.

In a discriminant analysis, a number of orthogonal, linear equations are derived of the following form:

$$
D=w_{1} V_{1}+w_{2} V_{2}+w_{3} V_{3}+w_{4} V_{4}
$$

where $w_{\mathrm{i}} \mathrm{s}$ are the calculated weights that maximize the discrimination between or among the groups based on $D$, and $V_{i} s$ are the predictor variables (in this experiment, $V_{i} s$ are the output activities of each of the four channels). In principle, the maximum

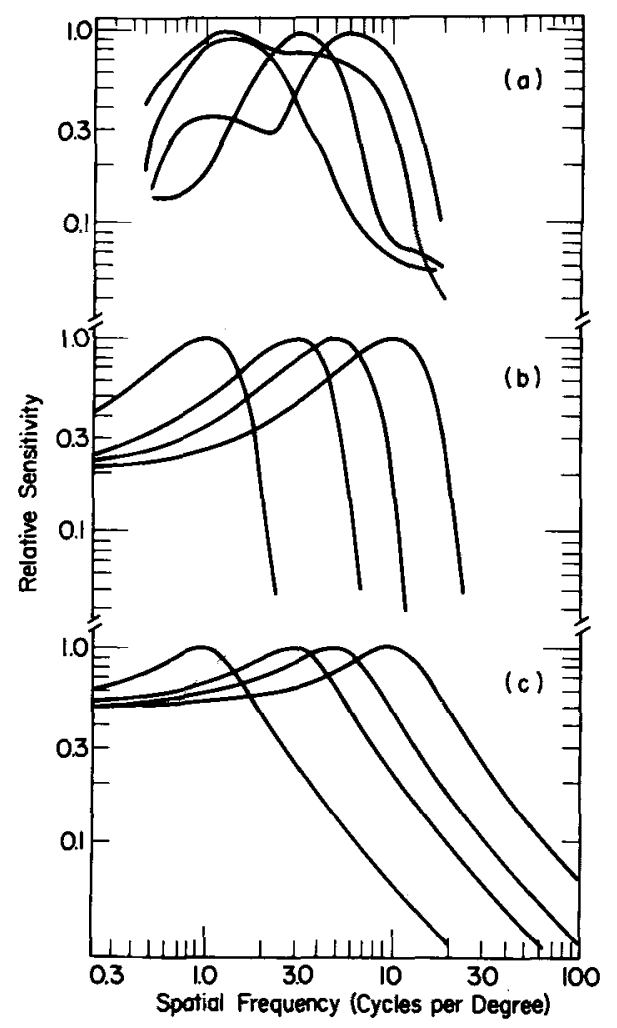

Figure 3. Characteristics of the three types of spatial-frequency channels modeled in our analysis. (a) Visibility, Jow-, medium-, and high-frequency channels of Richards and Polit (1974); (b) exponential filter channels from Mostafavi and Sakrison (1976); and (c) Butterworth filter channels from Mostafavi and Sakrison (1976). 
number of such LDFs that can be derived from a set of data is either one less than the number of groups formed or is equal to the number of predictor variables, whichever is smaller. Often the number of statistically significant LDFs found is smaller than the maximum number possible.

In order to assess the effectiveness of the discriminant analysis, we used a Monte Carlo procedure to provide a performance level based on chance against which to compare the groupings provided by our subjects. A computer program generated 40 different arrangements of the textures into two random groups which were then submitted to exactly the same discriminant analyses as were performed on the twogroup experimental data. In Table 2 , the experimental data are compared with the random data on four indices of the success of the discriminant analysis. The first index of success is the mean canonical correlation of the $\mathbf{4 0}$ experimental groupings compared with the mean canonical correlation of the 40 random groupings. The canonical correlation is a measure of the degree of the relationship between the groups, on the one hand, and the predictor variables, on the other. Table 2 shows that the mean canonical correlation is significantly higher for the experimental data than for the random data $(p<.0005$ for all three types of channels). The second index of LDF success is the percent of cases (out of 40 ) in which the LDF reached statistical significance at the .05 probability level. The channel models provided a far better prediction of the experimental data than of the random groupings. Using the Richards and Polit channels as predictor variables, for example, $72.5 \%$ of the 40 sets had significant LDFs while only $2.5 \%$ of them were significant with the random groupings. The third index is the ability of the LDF to classify the 30 stimulus textures into the correct group. Table 2 shows that the LDFs derived from the experimental groupings were significantly better than the LDFs derived from the random groupings $(p<.0005)$. The final index is $D$, the distance between the two group centroids, based on the LDF calculated for the groups. The better the LDF is at discriminating the two groups, the larger $D$ will be. The index $D$ is analogous to $d^{\prime}$ in signal detection theory. The mean value of $D$ is greater with LDFs calculated from the experimental groupings than with those based on the random groupings $(p<.0005)$.

Two other findings come out of this analysis. The first is that the Richards and Polit channels were slightly better on all four indices of performance than the other two types of channels (Table 2). The second finding concerns the number of significant discriminant functions found in the analysis of the 2, 3, 4, and 5 groupings. Although, in principle, the number of significant functions could have risen to four in the five-group analysis, it did not. Figure 4 presents the mean number of significant discriminant func-

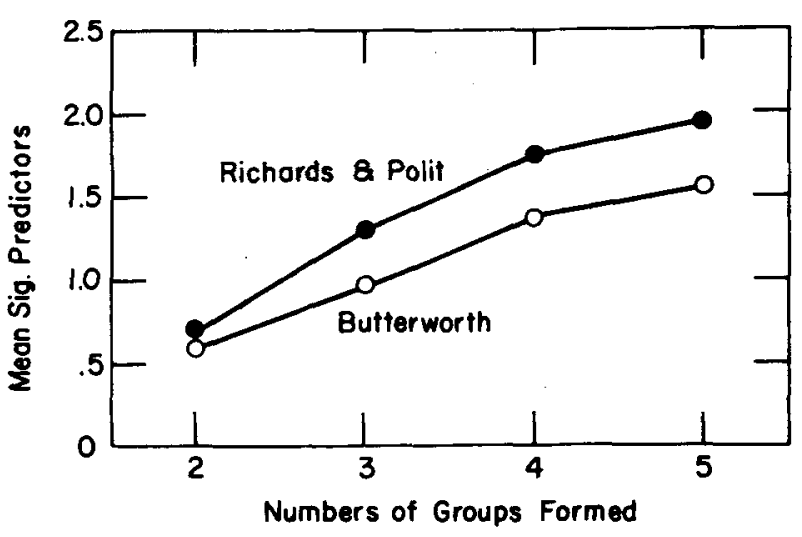

Figure 4. Mean number of significant linear discriminant functions (LDFs) as a function of number of groups formed by the subjects. Filled circles are LDFs based on Richards and Polit's (1974) channels and open circles are LDFs based on Butterworth filter channels.

tions calculated from analyses of the 2-, 3-, 4-, and 5-group data, for the Richards and Polit and the Butterworth filter channels. Notice that the Richards and Polit channels resulted in greater average number of significant LDFs than did the Butterworth channels. But more importantly, notice that the number of significant LDFs never exceeded two, even for the five-group analysis. Although there were four channels available to the subjects for making their groupings, they never used more than two independent combinations of the channels.

\section{Multidimensional Scaling}

A second, independent approach was taken to analyze these data. A 30 by 30 similarity matrix for the 30 textures was constructed based on the number of times a given texture pair was placed by subjects in the same group. Textures that are perceptually quite similar should almost always be placed in the same group (regardless of how the subject chose to configure the groups) and textures appearing quite dissimilar should almost never be placed in the same group. The similarity matrix was based on all the data: all 20 subjects, with two replications, with their $2,3,4$, and 5 groupings. Each stimulus pair had a maximum possible similarity score of 160 (if they were placed in the same group by all subjects on every task) and a minimum score of zero. The highest similarity score actually found in the experimental data was 148 and the lowest was 0 , indicating that our 20 subjects had been making very similar kinds of groupings.

The similarity matrix was analyzed using the nonmetric, multidimensional method developed by Kruskal (1964a, 1964b) and Shepard (1962a, 1962b). Version 5MS of the MDSCAL computer program was used (Kruskal \& Carmone, 1970). Six different 
Table 3

Factor Loadings, Eigenvalues, and Percent Variance Accounted for with Three Orthogonally Rotated Principal Components

\begin{tabular}{lccc}
\hline & \multicolumn{3}{c}{ Factor Number } \\
\cline { 2 - 4 } & \multicolumn{3}{c}{ Three-Dimensional Scaling } \\
\hline & .002 & .9998 & .019 \\
Dimension 1 & -.163 & .020 & .986 \\
Dimension 2 & .987 & .002 & -.163 \\
Dimension 3 & 1.324 & 1.000 & .676 \\
Eigenvalues & 44.1 & 33.3 & 22.5 \\
Percent Variance & Four-Dimensional Scaling \\
& .921 & -.267 & .131 \\
Dimension 1 & -.000 & -.137 & .989 \\
Dimension 2 & .008 & .979 & -.143 \\
Dimension 3 & -.905 & -.311 & .141 \\
Dimension 4 & 1.674 & 1.443 & .733 \\
Eigenvalues & 41.8 & 36.1 & 18.3 \\
Percent Variance & Richards \& Polit Channels \\
& .905 & .224 & .348 \\
Visibility Channel & .961 & -.259 & .033 \\
Low-Frequency Channel & .198 & .255 & .946 \\
Medium-Frequency Channel & .056 & .965 & .251 \\
High-Frequency Channel & .117 & 1.447 & 4.15 \\
Eigenvalues & 52.9 & 36.2 & 10.4 \\
Percent Variance & & &
\end{tabular}

runs were made, each with a different random starting configuration of the stimuli. Solutions were found for $6,5,4,3$, and 2 dimensions, using the Euclidean distance metric. The obtained stress values (a goodnessof-fit index) were identical on all six runs, so we concluded that local minima were not problems in the data. The stress values (Formula 2, Kruskal \& Carmone, 1970) for the different dimensionalities were: $6-\mathrm{D}=.0654 ; \quad 5-\mathrm{D}=.0777 ; \quad 4-\mathrm{D}=.0894$; $3-\mathrm{D}=.1122 ;$ and $2-\mathrm{D}=.3202$.

To help decide which dimensional scaling solution to accept, principal component factor analyses, with orthogonal varimax rotation of the axes, using 1.0 on the diagonals of the correlation matrices, were computed on the three- and the four-dimensional solutions (Table 3). When three factors were extracted, each of the three dimensions of the 3-D MDSCAL solution loaded highly on only one factor. The 3-D solution had a stress value of .1122 , which was considered good (Kruskal \& Carmone, 1970). Adding a fourth dimension to the MDSCAL solution improved the stress a bit (.0894), but did not change the number of factors extracted in the factor analysis. Table 3 shows that the fourth MDSCAL dimension has a high negative loading on the same factor (Factor 1), as does the first dimension, and that the other two dimensions have high loadings on separate factors. In addition, the three factors account for $96.2 \%$ of the variance in the four-dimensional solution. The three-dimensional MDSCAL solution is presented in Figure 5.
We are now in a position to represent the 30 stimulus textures as 30 points in two completely different, and independently derived, spaces: the threedimensional perceptual space from the MDSCAL analysis of the similarity matrix and the fourdimensional neural space based on the activity of the four spatial-frequency channels. If the perceptual appearance of the textures depends upon the activity of the four channels, then the configuration of the stimulus points in the perceptual space should be systematically related to the configuration of the same points in the neural space.

One statistical method for examining the relationship between the configuration of objects in two different spaces is canonical correlation analysis (Cooley \& Lohnes, 1971; van de Geer, 1971). The 30 textures are each represented by the activity evoked in the four Richards and Polit (1974) channels: $R P_{V}$, $R P_{L}, R P_{M}$, and $R P_{H}$. The 30 textures are each also represented by the three-dimensional MDSCAL configuration, $\mathrm{MD}_{1}, \mathrm{MD}_{2}$, and $\mathrm{MD}_{3}$. Canonical correla-
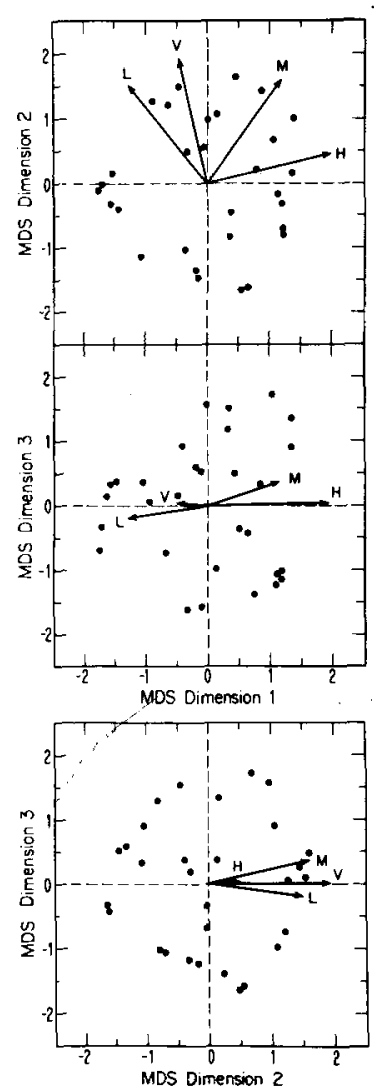

Figure 5. Three-dimensional MDSCAL stimulus configuration. Filled circles represent the $\mathbf{3 0}$ stimuli. The solid lines with arrowheads represent the best-fitting vectors of the visibility $(V)$, low(L), medium- (M), and high- (H) frequency channels of Richards and Polit (1974). 
tion analysis forms sets of two linear equations, called canonical variate pairs from these variables:

$$
\begin{gathered}
\alpha_{1}=a_{1} R P_{V}+a_{2} R P_{L}+a_{3} R P_{M}+a_{4} R P_{H} \\
\beta_{1}=b_{1} M D_{1}+b_{2} M D_{2}+b_{3} M D_{3}
\end{gathered}
$$

The analysis first calculates the numerical value of the weights $a$ and $b$ such that the Pearson product correlation between $\alpha_{1}$ and $\beta_{1}$ across the 30 stimuli is maximum. The value of this correlation is the canonical correlation. A geometrical interpretation of the canonical variate pair is that they are a pair of axes, one in each of the two spaces, that are maximally correlated. The analysis then calculates a second set of canonical variate pairs, $\alpha_{2}$ and $\beta_{2}$, orthogonal to the first set, in order to account for the variance unaccounted for by the first set. This procedure continues until either no further significantly correlated canonical variates can be extracted or the number of canonical variate pairs equals the dimensionality of the lowest dimensional space. In the above example, only three canonical variate pairs may be formed because the MDSCAL configuration contains only three dimensions.

The 30 stimuli as represented in the four-dimensional neural spaces of the Richards and Polit channels, of the Butterworth filter channels, and of the exponential filter channels were canonically correlated with the 30 stimuli, as represented by the 2-, 3-, 4-, 5-, and 6-dimensional MDSCAL configurations. These 15 separate canonical correlations were performed using SPSS (Nie et al., 1975). In each and every one of the 15 analyses, only two of the possible canonical correlations were statistically significant at the .05 level. These two canonical correlations for each of the analyses are given in Table 4. The total percentage of variance shared by the two separate representations (perceptual and neural) is calculated by summing the squared canonical correlations for each analysis and

Table 4

Canonical Correlations and Total Variance Explained Between

\begin{tabular}{|c|c|c|c|c|c|}
\hline & \multicolumn{5}{|c|}{ MDSCAL Dimensionality } \\
\hline & 2 & 3 & 4 & 5 & 6 \\
\hline & \multicolumn{5}{|c|}{ Richards \& Polit } \\
\hline $\begin{array}{l}\text { Canonical Correlation } 1 \\
\text { Canonical Correlation } 2\end{array}$ & $\begin{array}{l}.894 \\
.679\end{array}$ & $\begin{array}{l}.926 \\
.829\end{array}$ & $\begin{array}{l}.934 \\
.857\end{array}$ & $\begin{array}{l}.945 \\
.869\end{array}$ & $\begin{array}{l}.949 \\
.872\end{array}$ \\
\hline \multirow[t]{2}{*}{ Percent Variance } & 63.0 & 77.2 & 80.3 & 82.4 & 83.0 \\
\hline & \multicolumn{5}{|c|}{ Butterworth } \\
\hline $\begin{array}{l}\text { Canonical Correlation } 1 \\
\text { Canonical Correlation } 2\end{array}$ & $\begin{array}{l}.917 \\
.511\end{array}$ & $\begin{array}{l}.941 \\
.734\end{array}$ & $\begin{array}{l}.944 \\
.748\end{array}$ & $\begin{array}{l}.957 \\
.751\end{array}$ & $\begin{array}{l}.959 \\
.778\end{array}$ \\
\hline \multirow[t]{2}{*}{ Percent Variance } & 55.1 & 71.2 & 72.5 & 74.0 & 70.2 \\
\hline & \multicolumn{5}{|c|}{ Exponential } \\
\hline $\begin{array}{l}\text { Canonical Correlation } 1 \\
\text { Canonical Correlation } 2\end{array}$ & $\begin{array}{l}.904 \\
.539\end{array}$ & $\begin{array}{l}.930 \\
.770\end{array}$ & $\begin{array}{l}.932 \\
.784\end{array}$ & $\begin{array}{l}.947 \\
.786\end{array}$ & $\begin{array}{l}.951 \\
.804\end{array}$ \\
\hline Percent Variance & 55.4 & 72.9 & 74.2 & 75.7 & 77.5 \\
\hline
\end{tabular}
the MDSCAL Solutions and the Channel Outputs

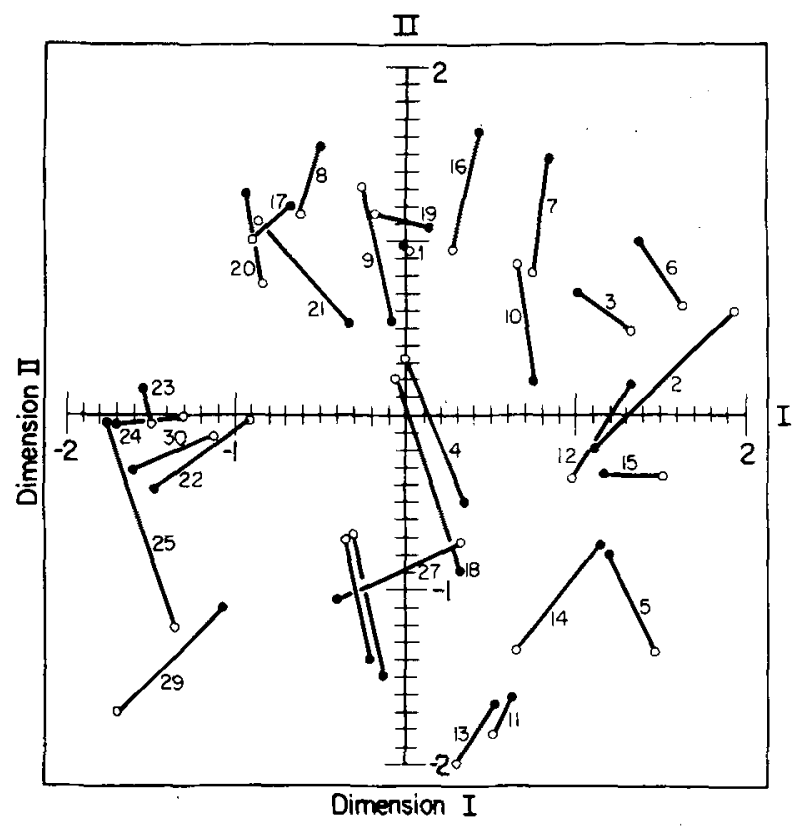

Figure 6. Two-dimensional plane of congruence between the spatial representations of the 30 stimuli based on the threedimensional MDSCAL solution (filled circles) and the configuration based on the activity of the four Richards and Polit channels (open circles). (Lines connect pairs of circles representing same texture.)

dividing by two. Table 4 , for example, shows that the Richards and Polit four-channel representation of the stimuli shares $77.2 \%$ of the variance with the threedimensional MDSCAL representation. Table 4 also shows that the Richards and Polit channels consistently accounted for more of the variance in the MDSCAL representations than did the other two types of channels.

Figure 6 represents the position of the 30 textures in a plane defined by the two significant sets of canonical variate pairs. Each texture is represented twice: once as a point whose $x, y$ coordinates are calculated by the first and second canonical variates $\alpha_{1}$ and $\alpha_{2}$, based on the four Richards and Polit channels (open circles); and again as a point whose $x, y$ coordinates are calculated by canonical variate sets $\beta_{1}$ and $\beta_{2}$, based on the three-dimensional MDSCAL solution (filled circles). The pairs of points in Figure 6 representing the same stimulus textures are connected by straight lines. If there were perfect congruence between the neural and the perceptual spaces, the filled and open circles would lie on top of each other. Were there no congruence between these two spaces, the lines would be random in length. The shorter the lines, the better the congruence. As given in Table 4, the congruence shown in Figure 6 represents canonical correlations of .926 and .829 , resulting in $77.2 \%$ total shared variance.

Both the discriminant analysis and the multidimensional scaling analysis suggest that there are only two 
independent factors relating the spatial-frequency channel activity to the perceptual appearance of the visual textures. A principal-component factor analysis with varimax rotation was computed for the Richards and Polit channels. The results are given in Table 3. There are only two factors with eigenvalues greater than 1.0 , and these account for $89.1 \%$ of the variance across the 30 stimuli. Adding the third factor (eigenvalue $=.415$ ) increases the variance accounted for to $99.5 \%$. After orthogonal rotation, the visibility and low-frequency channels load highly on Factor 1, the medium-frequency channel loads highly on Factor 3, and the high-frequency channel loads highly on Factor 2 (Table 3 ). Since the visibility and low-frequency channels were highly correlated in this stimulus set, one of them could have been eliminated from our analysis without much loss of information.

To clarify the relationship between the axes of the three-dimensional MDSCAL configuration and each of the four Richards and Polit channels, four multiple regressions were calculated using the three MDSCAL dimensions as predictor variables, one regression for each of the four channels. This analysis is equivalent to locating a direction vector through the 3-D MDSCAL space that maximally correlates with each of the four channels. The direction vectors for the visibility (V), low (L), medium (M), and high (H) spatial-frequency channels are drawn in the scaling solution shown in Figure 5. The $x, y$ coordinate of each vector tip in Figure 5 is the correlation of that channel (multiplied by 2.0 for graphic clarity) with the corresponding MDSCAL axis. Our previous conclusion that there were only two independent factors relating the channel activity to the perceptual appearance of the textures is clearly shown by the direction vectors in Figure 5. None of the vectors correlates with the third MDSCAL axis, leaving only Dimensions 1 and 2 related to the channels. Dimension 1 is most closely related to the vector of the high spatial-frequency channel $(\mathrm{H})$, while Dimension 2 is most closely related to the visibility channel (V). The remaining two channels, low (L) and medium (M) frequency, are related equally to Dimensions 1 and 2, but in an antagonistic manner.

\section{DISCUSSION}

The major conclusion of this study is that there is a systematic relationship between the spatialfrequency content of visual textures as represented by the activity evoked in four spatial-frequency channels and the perceived similarity of the visual textures. This conclusion is based on the ability of linear discriminant analysis to discriminate the perceptually similar groupings of the textures formed by our subjects using the activity of the four channels as predictor variables. It is further based on the observed congruence between the spatial representation of the stimuli based on judged similarity and the spatial representation of the stimuli based on channel activity.

The issue of dimensionality of the similarity space is an interesting one. Using a grouping task similar to the current experiment, but with eight-sided random polygons as stimuli (generated by Method 1 of Attneave \& Arnoult, 1956), Aiken and Brown (1971b) found an upper limit of three significant discriminant functions even when subjects wwere asked to form as many as nine groups (their predictor variables were geometric attributes of the polygons, not their spatial-frequency content). This same upper limit of three significant LDFs was also found by Aiken and Brown (1971a) when subjects were required to classify random polygons into two classes of patterns. A number of multidimensional scaling studies using random polygons as stimuli have concluded that the three-dimensional MDSCAL solution is the appropriate one (Aiken \& Brown, 1969a, 1969b, 1969c; Behrman \& Brown, 1968; Brown \& Andrews, 1968), although Stenson (1968) accepted a four-dimensional solution. Even with children as young as 7 years old, the three-dimensional MDSCAL solution is found to best describe similarity judgments (Aiken \& Williams, 1975). These results are consistent with Attneave's suggestion that all intellectual judgments, no matter how complex, are projected onto a "working space" within the mind (or brain) that is limited to three dimensions, and that this limitation is a consequence of our brain having evolved to deal with a three-dimensional world (Attneave, 1974). Our current data are consistent with this limit of three dimensions.

Figure 1 contains a schematic drawing of the postdetection processes that could construct an internal representation of the textures that would allow systematic judgments of visual similarity to be made. The outputs of the four spatial-frequency channels are combined to form two (or three) higher order channels. These new channels project onto a "working space"' in which each texture is represented as a point in the space. The distance between any two points represents the perceptual similarity of the corresponding two visual textures. In Figure 1, the pair of textures $\mathrm{A}$ and $\mathrm{B}$ would be judged more similar than pair $A$ and $C$ or $C$ and $B$ because of their relative separation in space. How, or even if, this schematic model is realized by neural mechanisms would be a matter of vigorous speculation, which for the moment we wish to avoid.

We do not want to conclude much about the specific channels modeled in this paper, except to say that all three of them gave good prediction of the experimental results. That Richards and Polit's (1974) four channels were consistently better than the other two by a small margin may have been due to the 
particular parameter values (peak frequencies and bandwidths) that were chosen. Rather than engage in a massive search for the parameter values that would have given the "best" fit with the data, we are content for the moment to conclude that a model of the visual system containing at least four spatialfrequency selective channels can account for much of the perceptual appearance of visual textures.

\section{REFERENCES}

Aiken, L. S., \& Brown, D. R. A spatial analysis of the discriminability of forms in noise. Perception \& Psychophysics, 1969, 5. 171-175. (a)

Aiken, L. S., \& Brown, D. R. Visual form perception: Congruence among spatial configurations. Perception \& Psychophysics, 1969, 5, 155-160. (b)

AIKEN, L. S., \& BRown, D. R. Visual form perception: Task demands and congruence among spatial solutions. Psychonomic Science, 1969, 15, 219-220. (c)

AIKen, L. S., \& Brown, D. R. A feature utilization analysis of the perception of pattern class structure. Perception \& Psychophysics, 1971, 9, 279-283. (a)

Aiken, L. S., \& BRown, D. R. Feature utilization of continuously varying attributes in visual pattern classification. Perception \& Psychophysics, 1971, 9, 145-149. (b)

Aiken, L. S., \& Williams, T. M. Development of multiple dimension use in form classification. Child Development, 1975, 46 , 123-132.

Attneave, F. How do you know? American Psychologist, 1974, 29, 493-499.

Attneave, F., \& Arnoult, M. D. The quantitative study of shape and pattern perception. Psychological Bulletin, 1956, 53, 452-471

BEHRMAN, B., \& BROWN, D. R. Multidimensional scaling of form: A psychological analysis. Perception \& Psychophysics, 1968, 4, 19-25.

Blakemore, C., \& Campbell, F. W. On the existence of neurones in the human visual system selectively sensitive to the orientation and size of retinal images. Journal of Physiology, 1969, 203, 237-260.

Brown, D. R., \& ANdrews, M. H. Visual form discrimination: Multidimensional analyses. Perception \& Psychophysics, 1968, 3, 401-406.

Campbell, F. W., \& Robson, J. G. Application of Fourier analysis to the visibility of gratings. Joumal of Physiology, 1968, 197, $551-566$.

COOley, W. W., \& Lohnes, P. R. Multivariate data analysis. New York: Wiley, 1971.

VAN DE GEER, J. P. Introduction to multivariate analysis for the social sciences. San Francisco: Freeman, 1971.

GRAHAM, N. Spatial frequency channels in the human visual system: Effects of luminance and pattern drift rate. Vision Research, 1972, 12, 53-68.

Graham, N., \& Nachmias, J. Detection of grating patterns containing two spatial frequencies: A comparison of single-channel and multiple-channel models. Vision Research, 1971, 11, 251-259.

Graham, N., \& Rogowitz, B. E. Spatial pooling properties deduced from the detectability of FM and quasi-AM gratings: A reanalysis. Vision Research, 1976, 16, 1021-1026.

HAKE, H. W., \& Rodwan, A. S. Perception and recognition. In J. B. Sidowski (Ed.), Experimental methods and instrumentation in psychology. New York: McGraw-Hill, 1966.

Henning, G. B., Hertz, B. G., \& Broadbent, D. E. Some experiments bearing on the hypothesis that the visual system analyzes spatial patterns in independent bands of spatial frequency. Vision Research, 1975, 15, 887-897.

KELLY, D. H. Spatial frequency selectivity in the retina. Vision Research, 1975, 15, 665-672.
Kelly, D. H. Pattern detection and the two-dimensional Fouriet transform: Flickering checkerboards and chromatic mechanisms. Vision Research, 1976, 16, 277-287.

Keliy, D. H., \& Magnuski, H. S. Pattern detection and the twodimensional Fourier transform: Circular targets. Vision Research, 1975, 15, 911-915.

KRUSKAL, J. B. Multidimensional scaling by optimizing goodness of fit to a nonmetric hypothesis. Psychometrika, 1964, 29. 1.27. (a)

Kruskal, J. B. Nonmetric multidimensional scaling: A numerical method. Psychometrika, 1964, 29, 115-129. (b)

KRuskal, J. B., \& Carmone, F. How to use MDSCAL [version 5M] and other useful information. Cambridge, Mass: Marketing Science Institute, 1970.

LEGGE, G. E. Adaptation to a spatial impulse: Implications for Fourier transform models of visual processing. Vision Research, 1976, 16, 1407-1418.

Mostafavi, H., \& SAKRIson, D. J. Structure and properties of a single channel in the human visual system. Vision Research, $1976,16,957-968$.

Nachmias, J., Sansbury, R., Vassilev, A., \& Weber, A. Adaptation to square-wave gratings: In search of the elusive third harmonic. Vision Research, 1973, 13, 1335-1342.

Nie, N. H., Hull, C. H., Jenkins, J. G., Steinbrenner, K., \& BENT, D. H. Statistical package for the social sciences (2nd ed.). New York: McGraw-Hill, 1975.

Quick, R. F., \& ReicherT, T. A. Spatial-frequency selectivity in contrast detection. Vision Research, 1975, 15, 637-643.

RIChards, W., \& Polit, A. Texture matching.Kybernetik, 1974, 16. $155-162$

RodWAN, A. S., \& HAKE, H. W. The discriminant-function as a model for perception. American Journal of Psychology, 1964, 77, 380-392.

Sachs, M. B., Nachmias, J., \& Robson, J. G. Spatial-frequency channels in human vision. Joumal of the Optical Society of America, 1971, 61, 1176-1186.

SCHNItZler, A. D. Theory of spatial-frequency filtering by the human visual system. I. Performance limited by quantum noise. Journal of the Optical Society of America, 1976, 66, 608-617. (a)

SCHNitzler, A. D. Theory of spatial-frequency filtering by the human visual system. II. Performance limited by video noise. Journal of the Optical Society of America, 1976, 66, 617-625. (b)

Sekuler, R. Spatial vision. Annual Review of Psychology, 1974, 25, 195-232.

SHEPARD, R. N. The analysis of proximities: Multidimensional scaling with an unknown distance function. I. Psychometrika, 1962, 27, 125-140. (a)

SHEPARD, R. N. The analysis of proximities: Multidimensional scaling with an unknown distance function. II. Psychometrika, 1962, 27, 219-246. (b)

SHEPARD, R. N. Representation of structure in similarity data: Problems and prospects. Psychometrika, 1974, 39, 373-421

SPITZBERG, R., \& RICHARDs, W. Broad band spatial filters in the human visual system. Vision Research, 1975, 15, 837-841.

Stenson, H. H. The psychophysical dimensions of similarity among random shapes. Perception \& Psychophysics, 1968, 3, $201 \cdot 214$.

Stromeyer, C. F., III, \& Julesz, B. Spatial-frequency masking in vision: Critical bands and spread of masking. Journal of the Optical Society of America, 1972, 62, 1221-1232.

Strome YeR, C. F, III, \& KLEIN, S. Evidence against narrow-band spatial frequency channels in human vision: The detectability of frequency modulated gratings. Vision Research, 1975, 15, 899-910.

Sullivan, G. D., Georgeson, M. A., \& Oatley, K. Channels for spatial frequency selection and the detection of single bars by the human visual system. Vision Research, 1972, 12, 383-394.

(Received for publication September 26, 1978; accepted October 12, 1978.) 